\title{
Real or imagined incomplete lateralization of function in females?
}

\author{
H. JULIA HANNAY \\ Auburn University, Auburn, Alabama 36830
}

\begin{abstract}
Visual field effects for 30 normal familial right-handed females were investigated with 4-point random forms known to be recognized more accurately in the right visual field by normal familial right-handed males, and the relationship between spatial ability and various performance patterns was examined. The forms were presented $2 \mathrm{deg}$ in the left or right visual field. After a 10-sec delay, the subject decided whether or not a form exposed in central vision was the same as the stimulus. Incomplete lateralization of function appeared to be supported by the finding of no field differences. An alternative explanation was discussed since visual field superiority was significantly correlated with WAIS Block Design scores scaled for age, females with relatively low scores showing a right visual field superiority and females with relatively high scores showing a left visual field superiority. The experiment was repeated with 30 familial right-handed males. A significant right visual field superiority was obtained, but visual field superiorities were not correlated with measures of visuo-spatial or verbal ability.
\end{abstract}

The concept of less complete lateralization of cerebral functions in females has received support from studies of both normal and brain-damaged subjects. It has been found, for example, that familial right-handed males show a significant right visual field superiority in the perception of verbal material (Hannay \& Malone, in press a) while familial right-handed females show only a slight right visual field superiority, if at all, for the same task (Hannay \& Malone, in press b). Similarly, a nonverbal task, dot enumeration, has produced a left visual field effect with a clear majority of right-handed males but with only about $50 \%$ of right-handed females (McGlone \& Davidson, 1973). Kimura (1969) found only a slight right visual field superiority for localization of dots in a square array by right-handed females in the presence of a significant left visual field superiority for right-handed males. Lansdell (1961) reported impairment of performance only for male patients on the Gorham Proverbs Test following left temporal lobe surgery. Analogously, Lansdell (1962) found that right temporal lobe surgery produced a performance decrement on the Graves Design Judgment only in male patients.

The main purpose of the experiment was to determine whether smaller field differences with females for memory of verbal stimuli (Hannay \& Malone, in press b) would obtain for the memory of forms that had previously produced a right visual field superiority with males. A task developed by Hannay, Rogers, and Durant (1976) and employing 4-point random forms was chosen to examine this possibility. Another purpose of this study was to determine

This research was supported by Research Grant-In-Aid 74-79 awarded by Auburn University. Thanks are extended to Maria Puente-Duany, Judith Rogers, and Dan Malone for collecting the data carefully and conscientiously. whether differences in visuo-spatial ability could account for some of the variability in performance patterns found among females on such visuoperceptive tasks. Accordingly, the Block Design Subtest of the Wechsler Adult Intelligence Scale (WAIS) was individually administered to each subject.

\section{EXPERIMENT I}

\section{Method}

Subjects. Thirty female undergraduates aged 18-26 years and en rolled in psychology courses at Auburn University received \$3 each for participation. All subjects classified themselves as strongly right-handed and stated use of their right hands for at least 8 of 10 activities listed on a modified version of the Neurosensory Center Handedness Questionnaire (Benton, Myers, \& Polder, 1962). All were familial right-handers having only right-handed parents and siblings.

Visual characteristics were determined with a Bausch and Lomb modified orthorater. Only subjects having $20 / 25$ or better acuity in each eye and both eyes together, normal vertical and lateral phoria, all at near and far distances, were included in the study.

Apparatus. A four-channel tachistoscope (Gerbrands Model T4A) with an automatic card changer and three semiautomatic card backs was employed. The fields were aligned and maintained at about $20 \mathrm{~cd} / \mathrm{m}^{2}$ as determined by an SEI photometer. The stimuli were presented in one field. The second field contained a small black circle and the third field, a small black dot, both centrally located on white cards. The fourth field contained a blank white card. The fields subtended $6^{\circ} 10^{\prime}$ of visual angle vertically and $8^{\circ} 50^{\prime}$ horizontally.

Stimuli. Random shapes (Vanderplas \& Garvin, 1959) of low complexity ( 4 inflexion points) and mean association value of 38.9 were selected. The practice stimulus was 7 and its incorrect foil (a fairly similar stimulus) was 26 . The pretest stimuli were $6,9,8$, and 13 , and the incorrect foils were $12,10,22$, and 14 , respectively. The test stimuli were $11,17,19,23,25,28$, and 30 , and the incorrect foils were $7,18,27,24,21,5$, and 20 . respectively. Outlines of the forms were drawn on white cards with India ink, beginning $2^{\circ}$ to the left or right of fixation. The forms subtended visual angles ranging from about $21^{\prime}$ to $55^{\prime}$ vertically and $14^{\prime}$ to $18^{\prime}$ horizontally.

Pretest task. The stimulus exposure duration was determined for each subject individually to produce the same level of performance 
for all subjects with 4-point stimuli presented in central vision. On cach trial. the subject tixated a small black circle in the center of the lighted screen. The black circle was replaced by a small black dot which served as a warning signal. Two seconds later. the dot disappeared and a form was presented in its place. The subject immediately looked to the left of the viewer where the experimenter was holding a card. The subject stated whether a form displayed on the card was exactly the same as (or differed from) the previously presented form. In a trial block. each of the four pretest stimuli was presented twice. once followed by a correct foil (a duplication of the stimulus) and once followed by its incorrect foil.

The pretest task consisted of five blocks of eight trials. The stimulus duration varied over trial blocks according to the rules of the block up and down two-interval forced choice procedure (Campbell. 1963). The tirst trial block constituted practice judgments and was ignored. A straight line relating the proportion of correct responses for the trial blocks and the respective stimulus durations was fitted to the data of the last four trial blocks by visual inspection. The duration expected to produce $75 \%$ correct responses in central vision was then determined. The starting duration for this procedure was found by increasing the exposure duration for a practice stimulus from $4 \mathrm{msec}$ by $2 \mathrm{msec}$ until the subject reported seeing a form after the black dot disappeared and stated that it differed from the incorrect practice foil displayed by the experimenter.

Test task. Preliminary testing suggested that it was necessary to add $4 \mathrm{msec}$ to the stimulus duration computed for each subject to maintain about $75 \%$ correct responses when the stimuli appeared in peripheral vision. As before. the subject fixated on a small black circle which was replaced by a small black dot. Two seconds later, the dot disappeared and a form was presented in left or right visual field. The subject continued to look at a blank white-lighted sereen until the circle reappeared $9 \mathrm{sec}$ after stimulus termination. The subject then made the same-different response to a foil. Since it takes about $1 \mathrm{sec}$ for the subject to look away from the viewer to the foil, a memory interval of about $10 \mathrm{sec}$ thus intervened between stimulus and response. Seven stimuli were presented four times in each visual tield. followed by the correct foil twice and by the incorrect foil twice for a total of 56 presentations.

The Block Design subtest of the WAIS was administered to each subject individually and a score scaled for age was determined.

\section{Results}

Subjects obtained a mean of $71 \%$ correct responses. which is fairly close to the desired level of $75 \%$. On the average. forms were recognized equally well in the left and right visual fields, $\mathrm{t}(29)=0.19$.
The mean number of correct responses was 18.93 in the right visual tield and 18.83 in the left visual field. WAIS Block Design scores scaled for age (mean = 11.7) were significantly correlated with the magnitude and direction of visual field differences, $\mathrm{r}(28)=.457$. $p<.02$. Fourteen subjects exhibited a right visual field superiority having a mean Block Design scaled score of 9.6 (range $=7-14$ ). This was significantly different, $\mathrm{t}(26)=4.95, \mathrm{p}<.001$, from the mean Block Design scaled score of 13.4 (range $=10-17$ ) for 14 subjects demonstrating a left visual field superiority. Two subjects showed no visual field superiority and had Block Design scaled scores of 11 and 17. The distribution of Block Design scaled scores and associated performance patterns is shown in Figure 1.

The mean stimulus duration was $22.4 \mathrm{msec}$ (range $=13-40 \mathrm{msec}$ ). The number of correct responses made by subjects was independent of stimulus duration, $\mathrm{r}(28)=.108$.

\section{Discussion}

Familial right-handed females showed no overall visual field difference for outline drawings of 4-point random forms. A lack of lateralization of cerebral functions and. in particular. linguistic functions would be an appropriate explanation for this finding since familial right-handed males had previously demonstrated a right visual field superiority with the same stimuli and task (Hannay, Rogers, \& Durant. 1976). But this inference is based on the averaged performance of many subjects and examination of individual differences in performance could lead to another explanation.

Retention of simple geometric forms could be mediated by verbal labels produced by the subject. Since the left hemisphere is likely to be dominant for linguistic functions in familial right-handers, verbal processing of this nature should result in a right visual tield superiority. Alternatively, a visual icon could

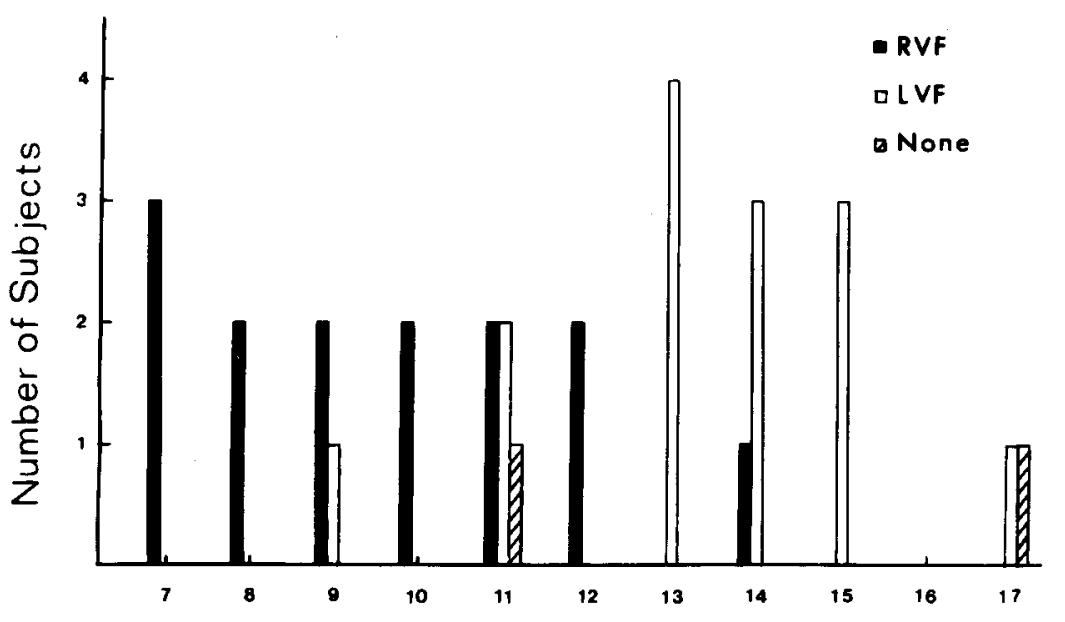

Block Design Scaled Score
Figure 1. Right visual field (RVF) and left visual field (LVF) superiorities for females as a function of Block Design scaled score. 
mediate retention of the forms. Such visuo-spatial processing should yield a left visual superiority for familial right-handers assumed to have righthemisphere dominance for visuo-spatial functions.

If subjects are divided into groups on the basis of visual field superiority and, by inference, mode of processing, an equal number of subjects processed the stimuli verbally as visuo-spatially. Females using the verbal mode of processing obtained relatively low Block Design scores which could be indicative of poor visuo-spatial ability and poor right-hemisphere functioning. Females employing the visuo-spatial mode of processing had relatively high Block Design scores. suggesting good visuo-spatial ability and good right-hemisphere function. Studies of sex-linked inheritance of visuo-spatial (Bock \& Kolakowski, 1973; Hartlage, 1970; Stafford, 1961) suggest that some females, though a lower percentage than males, should have good visuo-spatial ability. Thus, when the stimulus situation is ambiguous because the visually presented material can be processed verbally or visuo-spatially, visuo-spatial ability could be a major determinant of the mode of processing. The averaged performance of an equal number of females with relatively good and poor visuo-spatial ability could then be misinterpreted as no lateralization of visuo-spatial function. McGlone and Kertesz (1974) mention unpublished data of McGlone and Davidson (1973) which supports this contention. Females showing a right visual field superiority on a dot enumeration task tended to have lower scores on a test of visuo-spatial ability than females showing a left visual field superiority.

Why then did the majority of familial right-handed males previously process these forms verbally and not visuo-spatially (Hannay, Rogers, \& Durant, 1976) if more males have good right-hemisphere function than females? There is no ready answer to this question. No test of visuo-spatial ability was administered to the subjects in that study and one possibility is that the majority of those males had poor visuo-spatial ability, since the genetic model (Stafford. 1961) predicts that not all males should have good visuo-spatial ability.

McGlone and Kertesz (1974) have reported significantly lower Block Design scores for right-handed males with right- as compared to left-hemisphere disease but only slightly lower scores for right-handed females with right-hemisphere disease. Is Block Design performance a righthemisphere function in males but not in females? Our findings suggest that this is not so. Another interpretation of their data is, however, possible. If only a few women have good visuo-spatial ability and right-hemisphere function, the majority of women probably solve visuo-spatial tasks verbally and a significant decrement in performance would not be expected following right-hemisphere damage. Any decrement would be due to the lowered performance of those females with good visuo-spatial ability and right-hemisphere function. A visuo-spatial task, by definition, is a task which precludes or makes difficult the use of verbal mediation. Thus, females processing visuo-spatial tasks verbally might be expected to do no better than with the visuo-spatial mode of processing, if they had attempted it. In this case, left-hemisphere damage would not be expected to produce a greater deficit in performance for these individuals than right-hemisphere damage. When the left hemisphere was damaged, the task would be performed with the right hemisphere about as successfully as formerly with the left hemisphere.

\section{EXPERIMENT II}

The relationship between visuo-spatial ability and visual field effects for 4-point forms was investigated for familial right-handed males. The stimuli, apparatus, pretest, and test tasks were identical to those employed in the first experiment. Besides the WAIS block Design test of visuo-spatial ability, the Shipley-Hartford Vocabulary Test and a Controlled Word Association Test were administered to determine whether verbal ability might be related to field effects for 4-point forms also. For the Controlled Word Association Test, the subject gave all the words he could think of that began with the letters $P, R$, and $W$. One minute was allowed for each letter, and the total number of different words spoken by the subject was determined.

\section{Method}

Subjects. Thirty male undergraduates, aged 19-27 years and enrolled in psychology courses at Auburn University, participated for research credit. All subjects were strong familial right-handers meeting the criteria with respect to handedness and vision outlined in the first experiment.

\section{Results}

The mean number of correct responses was $67 \%$, which was somewhat lower than the desired level of $75 \%$, though not significantly so, $\chi^{2}(1)=3.41$, $\mathrm{p}>.05$. However, the number of correct responses was not related to stimulus durations, $r(28)=.093$, which ranged from 9 to $32 \mathrm{msec}$ (mean $=17.5 \mathrm{msec}$ ). Forms were recognized significantly more frequently in the right visual field than in the left visual field, $t(29)=1.77, p<.05$. The mean number of correct responses was 19.13 in the right visual field and 18.20 in the left visual field.

WAIS Block Design scaled scores (mean $=12.5$ ) were not a determinant of visual field superiorities, $r(28)=.273, p>.10$. Eighteen subjects showed a right visual field superiority and a mean Block Design scaled score of 12.8 (range $=10-17$ ), which did not vary significantly from the mean Block Design scaled score of 11.6 (range $=9-17$ ) for 11 subjects who 


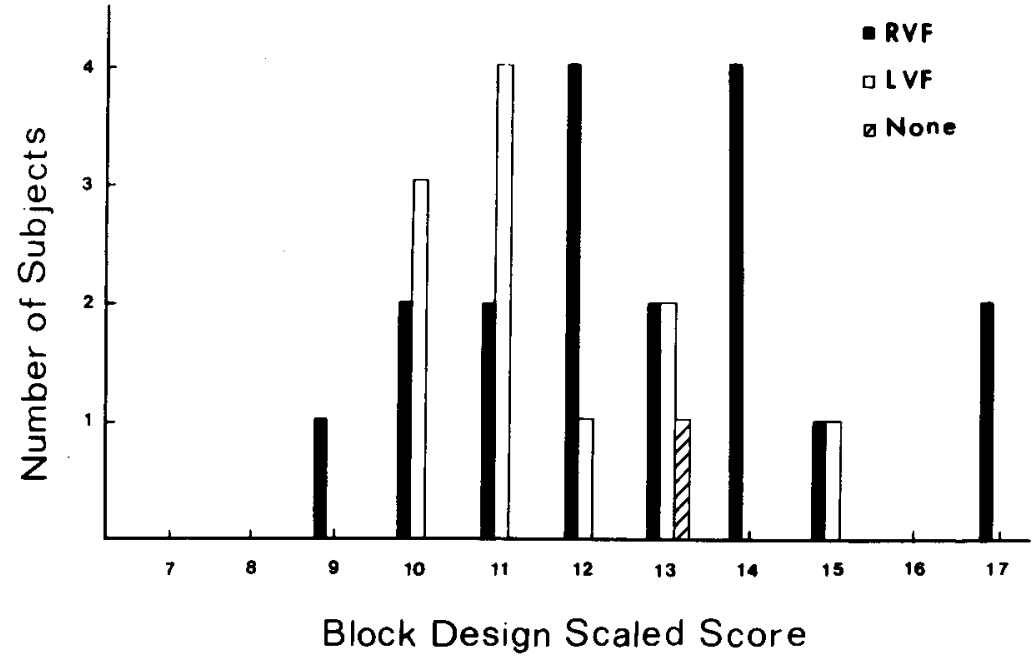

Figure 2. Right visual field (RVF) and left visual field (LVF) superiorities for males as a function of Block Design scaled score. showed a left visual field superiority, $t(27)=1.60$. $\mathrm{p}>.10$. Only one subject recognized the forms equally well in both visual fields having a Block Design scaled score of 13. The males in Experiment II had only slightly higher scores than the females in Experiment I. $\mathrm{t}(58)=1.27 . \mathrm{p}>.10$. ShipleyHartford Vocabulary scores were also not related to visual field superiority, $r(28)=.047$, nor were Controlled Word Association scores, $\mathrm{r}(28)=.022$.

\section{Discussion}

Previous research by Hannay, Rogers, and Durant (1976) was supported. Familial right-handed males showed an overall right visual field superiority for simple geometric forms. Since the average visuo-spatial ability of the males was not reliably different from that of the females, this right visual field superiority cannot be attributed to chance selection of males with relatively poor visuo-spatial ability. Furthermore, visuo-spatial ability was not a determinant of visual field superiorities. Males with high and low visuo-spatial ability obtained both right and left visual field superiorities.

Of course, there is no reason to assume that laterality effects are determined by individual differences in the same variable for males and females. Perhaps verbal ability controls the visual field superiority of males. This hypothesis was not supported either. At present, there seems to be no ready explanation for the disparate performance of males and females on this task.

The findings raise some interesting questions about the role of individual differences in abilities as determinants of visual field effects with tachistoscopic tasks. They also emphasize that more information about a subject's various abilities as well as sex, visual characteristics, handedness, and familial handedness is necessary for interpreting performance and may account for some of the variability in performance on visual laterality tasks.

\section{REFERENCES}

Benton. A. L.. Meyers, R.. \& Polder, G. Some aspects of handed ness. Psychiatn and Neurology, 1962, 144. 321-337.

Bock. R. D., \& Kolakowski, D. Further evidence of sexlinked major gene intluence on human visualizing ability. American Journal of Human Genetics. 1973, 25, 1-14.

CAMpBell. R. A. Detection of a noise signal of varying duration. Journal of the Acoustical Society of America, 1963, 35. 1732-1736.

Hannay, H. J., \& Malone, D. R. Visual field effects and short-term memory for verbal material. Neuropsychologia. in press. (a)

Hannay, H. J., \& Malone, D. R. Visual tield recognition memory for righthanded females as a function of familial handedness. Cortex. in press. (b)

Hannay. H. J.. Rogers. J. P., \& Durant. R. F. Complexity as a determinant of visual field effects for random forms. Actu Psychologica, 1976, 40. 29-34.

Hartlage, L. G. Sex-linked inheritance of spatial ability. Perceptual and Motor Skills, 1970. 31. 610.

Kimura. D. Spatial localization in the left and right visual field. Canadian Jnumal of Psychology, 1969. 23. 445-458.

LANSDELL. H. The effect of neurosurgery on a test of proverbs. American Psychologist. 1961, 16. 448.

LANSDELL. H. A sex difference in effect of temporal lobe neurosurgery on design preference. Nature, 1962, 194, 852-584.

McGlone. J., \& Davidson, W. The relation between cerebral speech laterality and spatial ability with special reference to sex and hand preference. Neuropsychologia. 1973. 11. 105-113.

McGlone. J.. \& Kertesz. A. Sex differences in cerebral processing of visuospatial tasks. Cortex, 1974, 10. 313-320.

StAFFord, R. E. Sex differences in spatial visualization as evidence of sex-linked inheritance. Perceptual and Motor Skills. $1961,13,428$.

Vanderplas. J. M.. \& Garvin, E. A. The association value of random shapes. Journal of Experimental Psychology, 1959, 57. 147-154.

(Received for publication December 8. 1975; revision received January $19,1976$. ) 